\title{
PHOTOELECTRON SPECTROSCOPY OF II-VI SEMICONDUCTOR HETEROSTRUCTURES*
}

\author{
M. Wörz, M. Hampel, R. Flierl and W. Gebhardt \\ Institut für Festkörperphysik, Universität Regensburg, Universitätsstr. 31, Germany
}

We are growing $\mathrm{ZnSe}, \mathrm{ZnS}$ and CdSe layers epitaxially on $\mathrm{GaAs}(001)$ substrates by atomic layer epitaxy and molecular beam epitaxy. The substrates are prepared by a H-plasma method in order to obtain a sharp interface between substrate and layer. The quality of our samples is controlled by reflection high energy diffraction and X-ray diffraction. Furthermore, the samples are characterized in situ by photoelectron spectroscopy. We observe resonant $\mathrm{Zn} 3 d^{8}$ and $\mathrm{Cd} 4 d^{8}$ satellites, which are used to check the layer quality. As a result, the valence band offsets of $\mathrm{CdSe} / \mathrm{ZnSe}$ and $\mathrm{ZnSe} / \mathrm{CdSe}$ were obtained. The values are $\Delta E_{\mathrm{v}}(\mathrm{ZnSe} / \mathrm{CdSe})=-(0.13 \pm 0.07) \mathrm{eV}$ and $\Delta E_{\mathrm{v}}(\mathrm{CdSe} / \mathrm{ZnSe})=-(0.13 \pm 0.07) \mathrm{eV}$, which confirm the commutativity rule.

PACS numbers: $79.60 . \mathrm{Jv}$

\section{Experiment}

$\mathrm{ZnSe}, \mathrm{ZnS}$ and CdSe layers are epitaxially grown on $\mathrm{GaAs}(001)$ substrates by molecular beam epitaxy (MBE), which is mostly used in the atomic layer epitaxy (ALE) mode. The quality of the surfaces is in situ controlled by reflection high energy diffraction (RHEED). In order to control structural properties of the samples high resolution X-ray diffraction is used. The valence band offset between these materials is determined by photoelectron spectroscopy (PES) with synchrotron radiation. A MBE-chamber was built, which has been attached to an analyzer chamber positioned at BESSY (Berliner Elektronenspeicherring-Gesellschaft für Synchrotronstrahlung $\mathrm{mbH}$ ). With this equipment heterostructures are grown in steps of monolayers and the surface is investigated during the growth interruptions in situ by UPS. In the ALE mode the different effusion cells are alternately opened. The successive openings of the cells containing the respective elemental IIand VI-materials is defined as one shuttercycle (SC). For the growth parameters see Ref. [1].

\footnotetext{
*This work was supported by BMBF 05 622WRA 0.
} 


\section{Results}

\subsection{Hydrogen plasma preparation}

The GaAs(001) substrates are cleaned by a chemical wet etching process [2]. A hydrogen plasma is used to remove the oxide overlayer. The substrate temperature is $200^{\circ} \mathrm{C}$. Figure 1 shows the spectra of a chemical etched GaAs(001) substrate without and with H-plasma treatment for various periods. Whereas in the first spectrum the oxide components of the core levels [3] and a strong oxide signal in the valence band dominate, these signals are obviously reduced after a 2 minutes' H-plasma treatment. A 4 minutes' treatment finally leads to a GaAs surface, where only the bulk components of the GaAs-core levels are seen but the oxide signal in the valence band is totally removed.

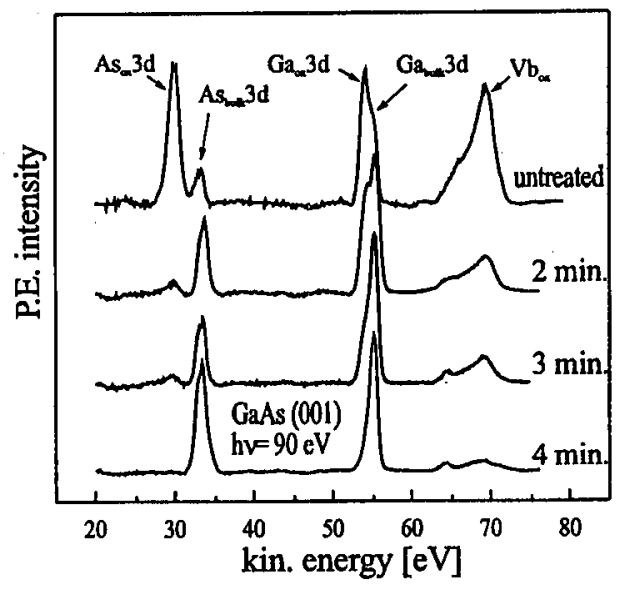

Fig. 1. UPS spectra of $\mathrm{GaAs}(001)$ before and after H-plasma treatment.

The preparation of $\mathrm{ZnSe}$ - and CdSe-surfaces leads to analogous results. The oxide components of the core levels are removed and the bulk components increase. A Se deficiency at the surface is observed, which completely vanishes after growth of a few $\mathrm{SC} \mathrm{ZnSe}$ or CdSe, respectively.

\section{2. $d^{8}$-satellites}

$\mathrm{GaAs}(001)$ substrates and $\mathrm{ZnSe}$ - or CdSe-epilayers are treated with hydrogen plasma. On these surfaces thin $\mathrm{ZnSe}$ - and CdSe-layers are grown and investigated with PES. Figure 2a shows different photoionization processes, which are especially relevant for the cases of $\mathrm{Zn} 3 d$ - and $\mathrm{Cd} 4 d$-levels. The transition $A$ describes the direct excitation of a $d$-shell electron into the continuum leading to a single hole $n d^{9}$ final state. $B$ is the MVV Auger process, $C$ is a non-resonant shake up and $D$ the resonant shake up. They all lead to two hole $n d^{8}$ final states. Furthermore, the photoexcitation $C$ and $D$ have the same final states and therefore give rise to resonant emission if the photon energy is near the $n p$ threshold. 

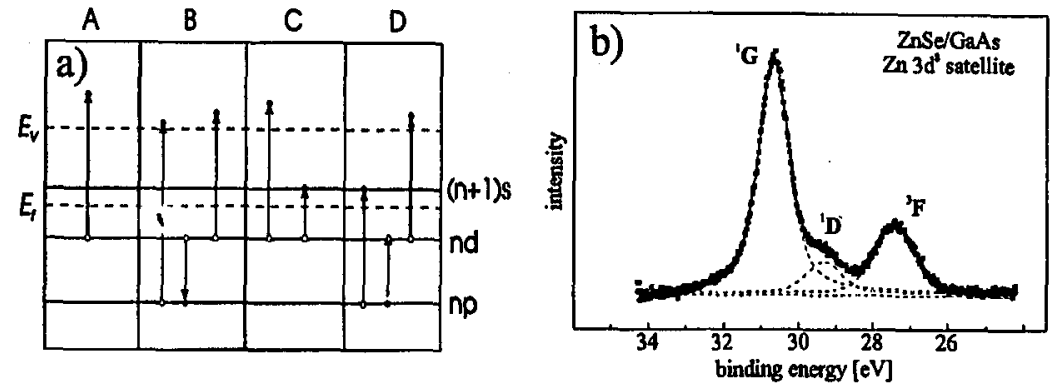

Fig. 2. (a) Excitation channels of a $n d^{10}$-core level, (b) $\mathrm{Zn} 3 d^{8}$-satellite in $\mathrm{ZnSe}$.

We write for the non-resonant shake up $(C)$

$\hbar \omega+n p^{6} n d^{10}(n+1) s^{0} \longrightarrow n p^{6} n d^{8}(n+1) s^{1}+\mathrm{e}^{-}$,

and for the resonant Auger $(D)$

$\hbar \omega+n p^{6} n d^{10}(n+1) s^{0} \longrightarrow n p^{5} n d^{10}(n+1) s^{1} \longrightarrow n p^{6} n d^{8}(n+1) s^{1}+\mathrm{e}^{-}$.

When the direct photoexcitation can be described by $E_{\text {kin }}=\hbar \omega-E_{B}-\phi$ ( $\phi$ - work function) and Koopman's theorem $E_{\mathrm{B}}=-\varepsilon_{j}\left(\varepsilon_{j}\right.$ - one-electron energy) is valid for the two hole final states $(B, C, D)$ the following relation holds:

$E_{\mathrm{B}}=-\left(\varepsilon_{j}+U_{\mathrm{eff}}\right), \quad U_{\mathrm{eff}}-$ effective Coulomb interaction.

Here the effective Coulomb interaction is the total additional energy, which is necessary to excite a second hole at the same atom and which deviates from two "one hole" energies. For a more detailed study of that process see Ref. [4].

Figure $2 \mathrm{~b}$ shows the $\mathrm{Zn} 3 d^{8}$-satellite observed at a photon energy of about $\hbar \omega \approx 90 \mathrm{eV}$. The multiplet splitting of the two-hole state should be the same for all Zn compounds, whereas the intensity ratio $I\left(d^{8}\right) / I\left(d^{9}\right)$ and the effective Coulomb interaction depend on the material (see Table).

TABLE

Characteristic results for the $\mathrm{Zn} 3 d^{8}$-satellite normalized to the Zn $3 d^{9}$-signal.

\begin{tabular}{l|c|c|c|c|c}
\hline \multicolumn{1}{c|}{ Compound } & $I\left(d^{8}\right) / I\left(d^{9}\right)$ & ${ }^{1} G[\mathrm{eV}]$ & ${ }^{1} D[\mathrm{eV}]$ & ${ }^{3} F[\mathrm{eV}]$ & $U_{\text {eff }}$ \\
\hline ZnSe (this work) & $16 \%$ & 21.5 & 20.2 & 18.2 & -12.3 \\
ZnS (this work) & $21 \%$ & 21.5 & 20.0 & 18.3 & -12.9 \\
Zn (metal) [5] & $6 \%$ & 22.3 & - & 16.8 & -10.3 \\
$\mathrm{ZnO} \mathrm{[6]}$ & $12 \%$ & 21.3 & 20.4 & 18.1 & -10.5
\end{tabular}

These two values should change if there are ternary regions in the grown heterostructure or if islands of the elemental materials are formed. We indeed observed a decrease in the intensity ratio $I\left(d^{8}\right) / I\left(d^{9}\right)$ at rough surfaces. Furthermore, while investigating this ratio for different photon energies it is possible to "scan" the $3 p$-level of $\mathrm{Zn}$ and to obtain the $3 p^{1 / 2}-3 p^{3 / 2}$ splitting. We find $\Delta E_{\mathrm{so}}(\mathrm{Zn} 3 p)=(3.03 \pm 0.05) \mathrm{eV}$. 
Analogous measurements have been performed at a photon energy of $\hbar \omega \approx$ $70 \mathrm{eV}$ for Cd $4 d$ in CdSe. We observed an intensity ratio $I\left(d^{8}\right) / I\left(d^{9}\right)=5 \%$, an effective Coulomb interaction $U_{\text {eff }}=-8.8 \mathrm{eV}$ and a Cd $4 p$-splitting $\Delta E_{\mathrm{so}}(\operatorname{Zn} 3 p)=$ $(7.54 \pm 0.05) \mathrm{eV}$.

\subsection{The valence band offset $\mathrm{ZnSe}-\mathrm{CdSe}$}

We have grown $\mathrm{ZnSe}$ on CdSe and CdSe on $\mathrm{ZnSe}$ in steps of a few monolayers and measured UPS spectra during growth interruptions to investigate the valence band offsets. The valence band edge is extrapolated by a straight line. Then the difference between the edge of the pure $\mathrm{ZnSe}$ and that obtained after growth of 70SC CdSe is determined. In the same way we treated the heterostructure $\mathrm{ZnSe} / \mathrm{CdSe}$. These differences have to be corrected for the band bending [7], which normally can be evaluated from the shift of the core levels. In the case of ZnSe-CdSe heterostructures, however, the only evaluable Se $3 d$ signal has to be fitted by a large number of different components (overlayer, CdSe, ZnSe, mixing components), which leads to a large uncertainty. For the Se $3 d$ core level of the pure materials we find $E_{\mathrm{B}}(\mathrm{Se} 3 d / \mathrm{ZnSe})=54.09 \mathrm{eV}$ and $E_{\mathrm{B}}(\mathrm{Se} 3 d / \mathrm{CdSe})=53.83 \mathrm{eV}$. These binding energies are determined as well for the heterostructure. By subtracting both core level differences we obtain the band bending.

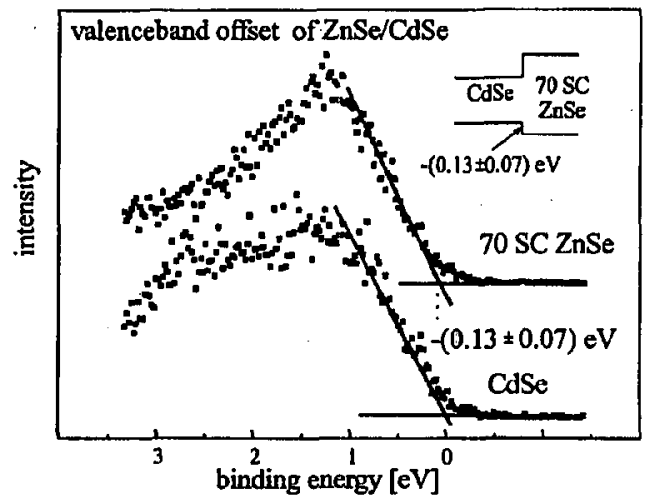

Fig. 3. The valence band offset at the interface of $\mathrm{ZnSe} / \mathrm{CdSe}$.

Figure 3 shows the valence band offset of $\mathrm{ZnSe} / \mathrm{CdSe}$ corrected by the band bending. The results for both directions $\mathrm{ZnSe} / \mathrm{CdSe}$ and $\mathrm{CdSe} / \mathrm{ZnSe}$ are

$$
\begin{aligned}
& \Delta E_{\mathrm{v}}(\mathrm{ZnSe} / \mathrm{CdSe})=-(0.13 \pm 0.07) \mathrm{eV} \text { and } \\
& \Delta E_{\mathrm{v}}(\mathrm{CdSe} / \mathrm{ZnSe})=+(0.12 \pm 0.07) \mathrm{eV} .
\end{aligned}
$$

It is obvious that in the case of $\mathrm{ZnSe-CdSe}$ heterostructures the commutativity rule is confirmed, which was found as well in $\mathrm{ZnSe}-\mathrm{ZnTe}$ [8]. These results agree well with results, which are derived from optical measurements of quantum wells by Lankes [9], who found $\Delta E_{\mathrm{v}}(\mathrm{ZnSe}-\mathrm{CdSe})= \pm(0.17 \pm 0.01) \mathrm{eV}$. 


\section{Acknowledgments}

We would like to thank the BESSY staff, Dr. K. Horn for providing us with his equipment at BESSY and Mr. G. Neuhold for his help with the analyzer.

\section{References}

[1] A. Rosenauer, T. Reisinger, E. Steinkirchner, J. Zweck, W. Gebhardt, J. Cryst. Growth 152, 42 (1995).

[2] S. Bauer, M. Huber, C. Rüth, P. Link, W. Gebhardt, Mater. Sci. Forum 143-174, 531 (1994).

[3] B.A. Cowans, Z. Dardas, W.N. Delgass, M.J. Carpenter, M.R. Melloch, Appl. Phys. Lett. 54, 365 (1989).

[4] L.J. Davies, J. Appl. Phys. 59, R29 (1986).

[5] M. Iwan, E.E. Koch, T.C. Chiang, F.J. Himpsel, Phys. Lett. A 76, 177 (1980).

[6] P.R. Bressler, Electronic Structure of Epitaxially Grown Magnetic Semiconductors on II-VI Compounds, Verlag Shaker, Aachen 1994.

[7] R.J. List, W.E. Spicer, J. Vac. Sci. Technol. B 6, 1228 (1988).

[8] P. Link, G. Grobbel, M. Wörz, S. Bauer, H. Berger, W. Gebhardt, J.J. Paggel, K. Horn, J. Vac. Sci. Technol. A 13, 11 (1995).

[9] S. Lankes, T. Reisinger, B. Hahn, C. Meier, M. Meier, W. Gebhardt, J. Cryst. Growth 159, 480 (1996). 